\title{
Towards the nasal delivery of insulin
}

"With several reports pointing to the figure of $17 \%$ bioavailability, the insulin delivery community needs to debate whether current nasal delivery technologies have evolved sufficiently to merit evaluation in the clinic."

Keywords: insulin $\approx$ mucoadhesion $₫$ nasal delivery

As the molecular design requirements for the preparation of nasally mucoadhesive polymers that are capable of combining the controlled and site-specific delivery of macromolecular therapeutic agents with penetration-enhancing properties are now reasonably well understood, the nasal delivery of insulin is fast becoming a technological reality.

\section{Nasal mucosa}

In common with other human mucosal membranes, those of the nose consist of a thin layer of differentiated epithelial cells, which are attached to the basement membrane that separates the epithelium from the underlying connective tissues. Because of the high packing density of basal cells within this membrane, a barrier is presented to microorganisms and also to drug absorption.

Research activities relating to therapeutic delivery through the nasal mucosa [1] are primarily driven by the need to develop drug-delivery strategies that avoid the first-pass metabolic effect, which impacts upon the bioavailability of many low-molecular-weight drugs and, more importantly, of macromolecular chemotherapeutants $[2,3]$. In addition, the high degree of vascularization, the thin epithelium and the large surface-to-volume ratio of the nasal cavity are all desirable features for efficient drug delivery.

\section{Absorption pathways}

The permeability of nasal mucosa is determined by the type of epithelial cells that cover the basement membrane. Although it is generally accepted that passive transport is the dominant mechanism for the transference of materials across mucosal membranes, the physicochemical characteristics of the permeant molecule may promote variable contributions from simple diffusion (transcellular and paracellular), carrier-mediated diffusion, active transport and pinocytosis or endocytosis [4]. For example, small lipophilic molecules favor transcellular transport, while charged hydrophilic macromolecules are normally transported paracellularly. Since tight junctions present the main barrier to the free flow of material from the apical lumen into the bloodstream, strategies for effecting the transient disruption of the conformation of junctional protein subunits are integral to the development of effective nasal formulations.

\section{Mucoadhesive-facilitated insulin delivery}

The efficacy of conventional dosage forms for the delivery of actives to the nasal cavity is often impeded by the residence time of the applied formulation. Common to all mucosal membranes is the barrier to absorption presented by the combined effects of the basement membrane, the associated lipophilic double layer and the overlying layer of mucus. Additional barriers to nasal delivery are presented by mucociliary clearance (approximately $4-6 \mathrm{~mm} / \mathrm{min}$ ), the relatively small volume of resident mucus that is available for mucoadhesive interactions $(100-500 \mu \mathrm{l}$; thickness of mucus blanket, approximately $5 \mu \mathrm{m}$ ), some nose-specific enzymatic activity and the high rate of mucus turnover (resting-state daily production, $75-150 \mathrm{ml}$ ). Consequently, over the past 40 years, there has been an increasing interest in the exploitation of the phenomenon of mucoadhesion (the adherence of a material to a mucosal surface for a period of time that extends beyond that of mucus turnover) for therapeutic benefits.

Since none of the theories that have been proposed to explain the process of mucoadhesion [5] can explain the behavior of all mucoadhesive formulations, some researchers have suggested that mucoadhesion progresses in stages, each of which is dominated by a different mechanism:

- Wetting and swelling of the dosage form (wetting theory);

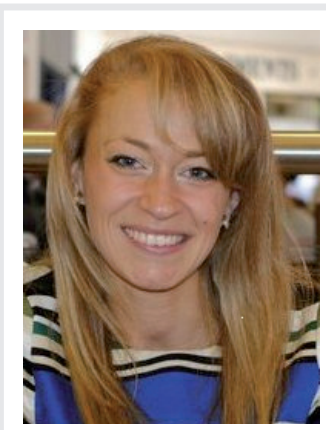

Hamde Nazar

Department of Pharmacy, Health \& Wellbeing, University of

Sunderland, Sunderland, Tyne \& Wear, SRI 3SD, UK

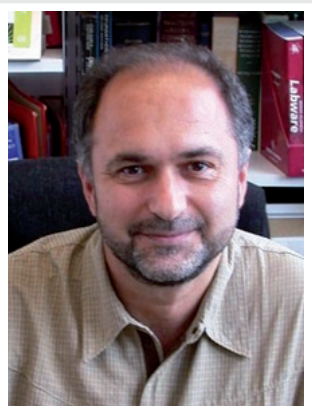

John Tsibouklis

Author for correspondence: Biomaterials \& Drug Delivery Research Group, School of Pharmacy \& Biomedical Sciences, University of Portsmouth, St Michael's Building, White Swan Road, Portsmouth, Hampshire POI 2DT, UK

Tel.: +442392 842131

Fax: +44 2392843565

E-mail: john.tsibouklis@port.ac.uk 
- Establishment of noncovalent, physical interactions at the mucus/polymer interface (electronic and adsorption theories);

- Interpenetrations of polymer and mucin molecules (diffusion theory);

- Entanglement and the formation of extended physical and covalent, chemical bondings (electronic and adsorption theories).

\section{Future perspective}

The considerable research activities towards the development of an intranasal system for the delivery of insulin are driven by the ongoing demand for increased ease of administration.

\section{"The considerable research activities towards the development of an intranasal system for the delivery of insulin are driven by the ongoing demand for increased ease of administration."}

The glucose-lowering effect of aqueous gel formulations of insulin and poly(acrylic acid) has been demonstrated (rat model) by Morimoto et al. [6]. Of considerable interest in the quest for the development of intranasal hydrogels for the delivery of insulin is chitosan [7-17]: a mucoadhesive polymer that is capable of effecting the transient opening of tight junctions via a mechanism that involves the translocation of occludin and ZO-1 from plasma membranes to the cytoplasm. The effectiveness of chitosan at opening tight junctions and its capacity for strong mucoadhesive interactions are both determined by the combined effects of concentration, molecular weight and degree of acetylation [10]. A thermosensitive in situ gelling nasal formulation of insulin, chitosan and poly(vinyl alcohol) has been shown to exhibit a prolonged hypoglycaemic effect, achieving a maximum reduction in blood glucose (approximately $40 \%$ ) at $4 \mathrm{~h}$ from administration as compared with a very similar (approximately $40 \%$ ) effect induced within $1 \mathrm{~h}$ by a subcutaneous insulin control [8]. Chung et al. have prepared an interpenetrating gel of glutaraldehyde-crosslinked chitosan, poloxamer and glycine that induces its maximum hypoglycaemic effect at approximately $5 \mathrm{~h}$ from administration [9]. The enhanced permeability imparted to nasal mucosa by thiolated or quaternised derivatives of chitosan, especially $N$-trimethyl chitosan chloride (TMC) $[10,18]$, has been demonstrated by du Plessis et al. [7]. The authors have reported that, as compared with a control solution of insulin, the nasal administration of a solution of insulin and highly quaternized (60\%) TMC effects a threefold increase in the intranasal absorption of insulin.

In view of the promise of TMC as a carrier matrix for the intranasal delivery of insulin, we have recently formulated an in situ thermogelling hydrogel for application as intranasal solution. This formulation effects a prolonged hypoglycaemic effect that is comparable with that reported by Barichello et al. [19] for the subcutaneous administration into healthy rats of insulin in Pluronic F127 gels or in gels containing insulin-PGLA nanoparticles (respective serum glucose levels $\left[\mathrm{C}_{\text {min }}\right]$ at approximately $60 \%$ of basal at $6 \mathrm{~h}$ from administration and of approximately $40 \%$ at $3 \mathrm{~h}$ ). Calculated with reference to the subcutaneous injection group (pharmacodynamic availability, $\left[\mathrm{F}_{\mathrm{dyn}}\right]$, of $100 \% ; 1 \mathrm{IU} / \mathrm{kg}$ ), the $\mathrm{F}_{\mathrm{dyn}}$ of insulin that had been delivered intranasally via this hydrogel (approximately 17\%) compares favourably with those reported for the administration of insulin via chitosan/hydroxypropyl-betacyclodextrin solution (16\%) [17], interpenetrating gel (18\%) [91], chitosan/PVA gel (9\%) [4] or derivatized chitosan (HTCC)-PEG hydrogel (7\%) [11].

While the evidence is that the pharmacodynamic availability of insulin from nasal delivery systems is limited to approximately $17 \%$, in vivo data are derived from the rat model with only a single report of an experiment in sheep [20] and with no published studies involving humans. With several reports pointing to the figure of $17 \%$ bioavailability, the insulin delivery community needs to debate whether current nasal delivery technologies have evolved sufficiently to merit evaluation in the clinic.

\section{Financial \& competing interests disclosure}

The authors have no relevant affiliations or financial involvement with any organization or entity with a financial interest in or financial conflict with the subject matter or materials discussed in the manuscript. This includes employment, consultancies, honoraria, stock ownership or options, expert testimony, grants or patents received or pending, or royalties.

No writing assistance was utilized in the production of this manuscript. 


\section{References}

1 Prego C, Garcia M, Torres D, Alonso MJ. Transmucosal macromolecular drug delivery. J. Control. Release 101(1-3), 151-162 (2005).

2 Luppi B, Bigucci F, Cerchiara T, Zecchi V. Chitosan-based hydrogels for nasal drug delivery: from inserts to nanoparticles. Expert Opin. Drug Deliv. 7(7), 811-828 (2010).

3 Duan XP, Mao SR. New strategies to improve the intranasal absorption of insulin. Drug Discov. Today 15(11-12), 416-427 (2010).

4 Blanchette J, Kavimandan N, Peppas NA. Principles of transmucosal delivery of therapeutic agents. Biomed. Pharmacother. 58(3), 142-151 (2004).

5 Khutoryanskiy VV. Advanced in mucoadhesion and mucoadhesive polymers. Macromol. Biosci. 11, 748-764 (2011).

6 Morimoto K, Morisaka K, Kamada A. Enhancement of nasal absorption of insulin and calcitonin using polyacrylic acid gel. J. Pharm. Pharmacol. 37, 134-136 (1985).

7 du Plessis L, Kotze A, Junginger H. Nasal and rectal delivery of insulin with chitosan and $N$-trimethyl chitosan chloride. Drug Deliv. 17, 399-407 (2010).
8 Agrawal AK, Gupta PN, Khanna A et al. Development and characterisation of in situ gel system for nasal insulin delivery. Pharmazie 65, 188-193 (2010).

9 Chung T-W, Liu D-Z, Yang J-S. Effects of interpenetration of thermosensitive gels by cross-linking of chitosan on nasal delivery of insulin: in vitro charactersation and in vivo study. Carbohyd. Polym. 82, 316-322 (2010).

10 Luppi B, Bigucci F, Cerchiara T, Zecchi V. Chitosan-based hydrogels for nasal drug delivery: from inserts to nanoparticles. Expert Opin. Drug Deliv. 7, 811-828 (2010).

11 Wu J, Wei W, Wang L-Y, Su Z-G, Ma G-H. A thermosensitive hydrogel based on quaternized chitosan and poly(ethylene glycol) for nasal drug delivery system. Biomaterials 28, 2220-2232 (2007).

12 Varshosaz J, Sadrai H, Heidari A. Nasal delivery of insulin using bioadhesive chitosan gels. Drug Deliv. 13, 31-38 (2006).

13 Rao SB, Sharma CP. Use of chitosan as a biomaterial: studies on its safety and hemostatic potential. J. Biomed. Mater. Res. 34, 21-28 (1997).

14 Chen R, Chen HC. Chitin enzymologychitinase. Adv. Chitin Sci. 3, 16-23 (1998).
15 Muzzarelli C. Human enzymatic activities related to the therapeutic administration of chitin derivatives. Cell. Mol. Life Sci. 53, 131-140 (1997).

16 Hirano S, Seino H, Akiyama Y, Nonaka I. Biocompatibility of chitosan by oral and intravenous administrations. Poly. Eng. Sci. 59, 897-901(1988).

17 Lehr C-M, Bouwstra JA, Schacht EH. In-vitro evaluation of mucoadhesive properties of chitosan and some other natural polymers. Int. J. Pharm. 78, 43-48 (1992).

18 Nazar H, Fatouros DG, van der Merwe SM et al. Thermosensitive hydrogels for nasal drug delivery: the formulation and characterisation of systems based on $N$-trimethyl chitosan chloride. Eur. J. Pharm. Biopharm. 77, 225-232 (2011).

19 Barichello JM, Morishita M, Nagai KTT. Absorption of insulin from Pluronic F-127 gels following subcutaneous administration in rats. Int. J. Pharm. 184, 189-198 (1999).

20 Dyer AM, Hinchcliffe M, Watts P et al. Nasal delivery of insulin using novel chitosan based formulations: a comparative study in two animal models between simple chitosan formulations and chitosan nanoparticles. Pharm. Res. 19, 998-1008 (2002). 